\title{
The EQ-5D-5L Valuation Study in Egypt
}

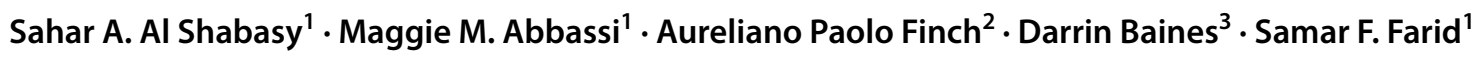

Accepted: 23 January 2021 / Published online: 12 March 2021

(c) The Author(s) 2021

\begin{abstract}
Introduction No value sets exist for either the EQ-5D-3L or the EQ-5D-5L in Egypt, despite 1 cal pharmas.coeconomic guidelines recommending the use of the EQ-5D to derive utility. Most published Egyptian ecorom vvaluation studies have used utility values from other published studies and systematic reviews.

Objective Our objective was to develop an Egyptian EQ-5D-5L value set using the $\mathrm{i}$ ternatio. EuroQol standardized protocol (EQ-VT-2.1).

Methods Adult Egyptian participants were recruited from public places using malti-strat $\mathcal{C}$ quota sampling based on age, sex, and geographical distribution. Two elicitation techniques were appli t: th composite time trade-off (cTTO) and discrete-choice experiments (DCEs). Before actual data collection, interviewers rromance was assessed in a pilot phase. Data were modelled using generalized least square, Tobit, heteroskeda tic, logit, 4 hybrid models, and the best fitting model was selected based on the value range between observed and predicta parameters, significance level, and prediction accuracy.

Results A total of 1378 interviews were conducted, of which 1 vere exc/uded because they were incomplete or did not comply with protocol, 216 were pilot interviews, and 974 re in ded in the final analysis. The heteroskedastic model (model 4) based on the cTTO data was selected as the pr ferre od / to generate the value set. Values ranged from -0.93 for the worst health state (55555) to 1 for full health 111), wit $1136(36.3 \%)$ of all predicted health states being worse than dead. Mobility had the largest impact on hea' $15 \mathrm{st}$. preff ence values.

Conclusion This is the first value set for the EQ D-5L bas on social preferences obtained from a nationally representative sample in Egypt or any Arabic-speaking country. e value set can be used as a scoring system for economic evaluations and to improve the quality of health tech Kology asses, ment in the Egyptian healthcare system.
\end{abstract}

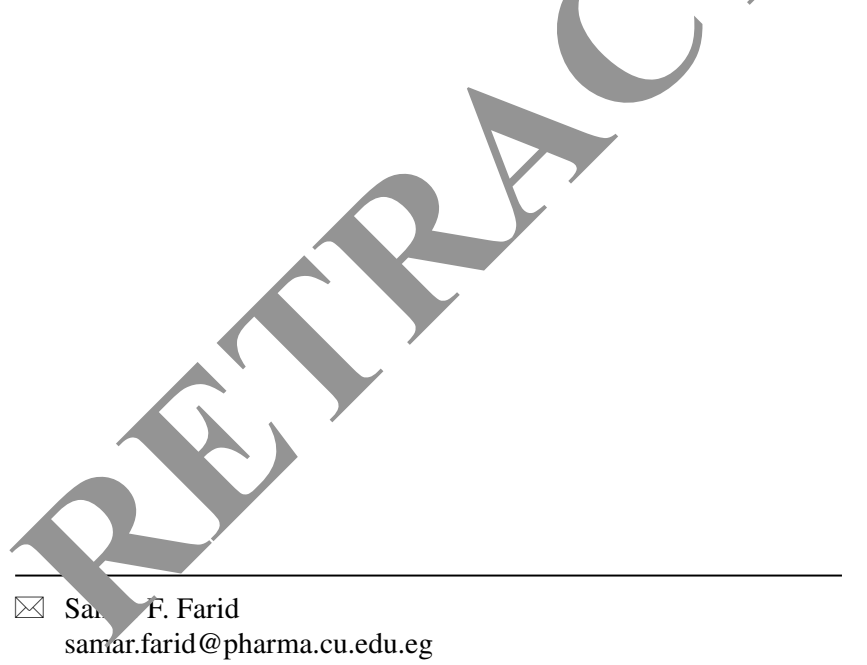

1 Department of Clinical Pharmacy, Faculty of Pharmacy, Cairo University, Kasr El-Aini St., Cairo 11562, Egypt

2 EuroQol Group Office, Rotterdam, The Netherlands

3 Business School, Dorset House Talbot Campus, Bournemouth University, Fern Barrow, Poole, UK

\section{Key Points for Decision Makers}

This is the first EQ-5D-5L valuation study in Egypt and in the Middle East and North Africa region.

The Egyptian tariff can be used as a scoring system for economic evaluations, to inform decision making, and to improve the quality of health technology assessment in the Egyptian healthcare system.

The availability of the Egyptian tariff will encourage health economists and clinicians to include quality-oflife questionnaires in clinical trials and implement costutility analysis and pharmacoeconomic modelling. 


\section{Introduction}

The EQ-5D is the most widely used preference-based health-related quality of life (HRQoL) measure, developed by the EuroQol Group [1]. It is used to inform resource allocation decisions in economic evaluations across the world [2-4]. In addition, it is the multi-attribute utility instrument preferred by most published pharmacoeconomic guidelines [5]. The EQ-5D consists of five dimensions: mobility, self-care, usual activities, pain/discomfort, and anxiety/depression. There are several versions: the three-level EQ-5D (EQ-5D-3L) defining 243 health states, the five-level EQ-5D (EQ-5D-5L) defining 3125 health states, and the youth version (EQ-5D-Y) used for pediatric populations $[6,7]$. The EQ-5D-5L has advantages over the EQ-5D-3L as it has more discriminatory power and even distribution with reduced ceiling effects [8-10].

Egypt is the most populous country in the Middle East, having a population of 100 million citizens [11], and exerts significant cultural influence on the region [12]. In Egypt, there is growing awareness of the importance of pharmacoeconomics. There is a great need to conduct high-quality economic evaluations to support and inform pricing and reimbursement decisions and to develop preference-based measures in different disease states. In Egypt, no value sets exist for either the EQ-5D-3L or the EQ-5D-5L; however, local pharmacoeconomic guidelines recommend the of the EQ-5D as one of the preferred methods to rite utility [13]. Most published Egyptian economi eva tion studies depend on utility values from oth ublishe studies and systematic reviews without a re ere value set for Egypt [14-19].

The aim of this study was to deve op the FQ-5D-5L value set for Egypt by eliciting general hlic rieferences, which will allow the assessmen f healtncare interventions using cost-utility analysis ine-s ss-country comparison of health technolos. asses ment (HTA) evidence.

\section{Methor's}

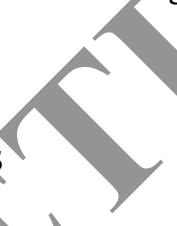

\subsection{Stua 'esig}

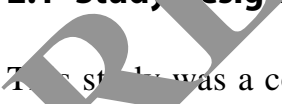

a computer-based, cross-sectional, interviem administered face-to-face survey of a representative Egyptian population following the EQ-VT developed for the valuation of the EuroQoL family of instruments [20]. This study was approved by the Research Ethics Committee at the Faculty of Pharmacy, Cairo University. Written informed consent was obtained from all participants. For reporting the key elements of the Egyptian valuation studies, we followed the CREATE checklist for multi-attribute utility instruments [21].

\subsection{The EQ-5D-5L Descriptive System}

The EQ-5D-5L describes five dimensions. Each dimension describes five levels of severity: no, mild, moderate, severe, and unable/extreme [2, 4]. The combination of the five dimensions and their levels results in a health ate. Each health state can be described by a five-digit nt. hoth t ranges from 11111 (no problems in any of the five $\mathrm{m} n$ sions) to 55555 (extreme problems or una to in a' dimensions). The level of sum scores or tic 'mu v score' is a proxy for severity and is calcula d by sumping the five digits for the given health state [2]

\subsection{Preference-Elicit- cion Te, niques}

The EQ-VT desion elicits ferences using the composite time trade- ff (c TO) and discrete-choice experiments (DCEs). The c. cowsts of the conventional TTO for health stotes bette. 'an dead and the lead-time TTO for states consin worse than dead. The cTTO design consists of a s. of 86 health states assigned to ten blocks. As the DCl/tasks, the participants are asked to choose bet en two impaired health states. It includes 196 pairs EC - $5 \mathrm{D}-5 \mathrm{~L}$ health states divided into 28 blocks of seven p. s. Detailed descriptions of the valuation protocol and the wo elicitation techniques have been previously published [20, 22-24].

\subsection{Sampling Method and Study Population}

Egypt is divided into seven regional units containing 27 governorates [25]. For the best geographical, social, and cultural representation, adult Egyptian participants were recruited from different Egyptian governorates representing all geographical areas as per the population distribution. Participants were recruited through personal contact and from public places such as university campuses, governmental authorities, sporting clubs, and shops using multi-stratified quota sampling based on Egypt official statistics updated in March 2019 [11], to select a representative sample in terms of age, sex, and geographical distribution. Adult participants who agreed to give informed consent and were able to understand the valuation tasks were included in the study. The interviews took place at the interviewer's office, or the participant's workplace or home, or other public places according to participants' preferences. The participants did not receive any incentives.

The interviewer team included 12 interviewers (11 females and one male). All interviewers were teaching assistants in the Clinical Pharmacy Department, Faculty of 
Pharmacy, Cairo University, who received intensive training by the research team using the training material received from the EuroQol. Egypt employs no sex segregation in education, work, or social interactions, so sex matching of interviewers and participants was not necessary.

\subsection{Pilot Phase}

A well-defined pilot phase ( $n=216$ interviews) took place from July to October 2019. The main objective of the pilot phase was to test the feasibility and cultural appropriateness of the EQ-VT protocol and to describe which specific elements of the protocol might need adaptation. Other objectives were to standardize interviewers' performance to reduce variability within and across interviewers, promote quality, and improve data distribution while avoiding clustering at specific values. Some adaptations were applied to the standard valuation protocol and the interview process where the initial practice health state 'wheelchair (WC) example' was changed to 'migraine example' as most of the participants stated that being in a WC would be worse than being dead. The WC example was originally designed to elicit a 'better than dead' response, so this change had the positive effect of ensuring consistency with other valuation studies, where the structure of the familiarization session remained unchanged with the application of the same quality control (QC) criteria. In addition, to facilitate illiterate participants' comprehension of the tasks, we used visual aids that were tested in the pilot phase. Graphics were used to represe. five dimensions, and colored cards (green, yell v, orang red, and dark crimson) were used to repres it lo ${ }^{1} \mathrm{~s} 1-5$, respectively. These colors were adapted from the trafi. ilght system familiar to participants. Intervie ers were instructed to read aloud the health states to illitera articipants twice while placing the colored cards ce responamg to the level of severity in front of the graphics to e $e_{\lambda}$ the health states as they appeared on the sc. All nethodological changes undertaken to accomm tate mitura and social considerations will be presen $\mathrm{d} d$ in ubsequent publication.

\subsection{Interview Pro -s}

The valua rasi sere carried out using the standardized ptia rabic version of the EQ-VT software (2.1), $\checkmark$ re articipants were given the study objectives with the clan ation that valuation tasks were not intended to cause any co $A$ iict with their spiritual or religious beliefs [20]. Participants then reported and rated their own health using the EQ-5D-5L descriptive system and visual analogue scale (VAS). Five practice cTTO tasks, followed by the valuation of ten cTTO hypothetical EQ-5D-5L health states, were then completed. Afterwards, a feedback module was completed in which the ten health states were arranged on the screen with the highest value at the top and the lowest value at the bottom, according to the participant's choices. Participants could flag any health state that was out of order (flagged health states were excluded from the final data analysis). Next, seven forced-pair comparison DCE tasks were presented in random order. Finally, participants completed a validated country-specific questionnaire pertaining to participants' views about health, life, and death.

For all the valuation tasks, participants were instructed to read the description of each health state alou chsule their engagement.

\subsection{Quality Control}

The EuroQol group developed a $\mathrm{C}$ tool $/ 26]$ to improve protocol compliance. This o ol ood interviews that were completed in less nan 3 in for the WC example or less than $5 \mathrm{~min}$ for th to. TTO tasks, interviews where the interviewer did not explain $\%$ 'worse than dead' (WTD) element of the $4 \mathrm{sk}$, interviews with clear inconsistencies. The QC tool at aum ed the presence of interviewers' effects by compar the distribution of cTTO data across interview any skewed distributions or spikes at -1 , $-0.5,0,0.5$, and 1 . The tool also detected any unusual pats in DC/Z responses. QC meetings were held between the yptian team and the EQ-VT support team, weekly the pilot phase and biweekly during actual data collecth , to discuss the QC reports. Interviewers were dropped or retrained based on their performance according to the $\mathrm{QC}$ reports.

\subsection{Data Analysis and Model Selection}

We used SPSS software version 22 for the descriptive statistics of the sample characteristics and responses to cTTO and DCE data, and statistical modelling was conducted using STATA software version 14 to estimate the EQ-5D-5L values for all health states. Several models were tested, including generalized least square (GLS), Tobit, heteroskedastic, conditional logit, and hybrid models. The 20-parameter model is a main-effect model consisting of 20 dummies, one for each dimension level from mobility level 2 to anxiety/depression level 5 (MO2-AD5) using level 1 as the reference. For the cTTO data, random effects (GLS) models (models 1,2, and 3) were tested to account for the panel structure of the data and heterogeneity of the participants' views in valuing EQ-5D-5L health states. Tobit models (models 2 and 3 ) were used to account for the censored nature of cTTO data because participants could hypothetically continue trading below the left lower bound at -1 for the WTD health states. The heteroskedastic models (models 3 and 4) were investigated to deal with the heteroskedasticity of the error term as the observed variance of the cTTO 
Table 1 Background characteristics of the Egyptian participants

\begin{tabular}{|c|c|c|c|c|}
\hline Characteristics & Full sample $(n=1303)$ & Actual sample $(n=974)$ & $\begin{array}{l}\text { General } \\
\text { population }^{\text {a }}\end{array}$ & $p$ value \\
\hline \multicolumn{5}{|l|}{ Sex } \\
\hline Male & $672(51.6)$ & $510(52.4)$ & 51.6 & 0.617 \\
\hline Female & $631(48.4)$ & $464(47.6)$ & 48.4 & 0.617 \\
\hline Age (years) & $35.8 \pm 12.8(18-75)$ & $36.9 \pm 12.7(18-72)$ & - & - \\
\hline $18-24$ & $317(24.3)$ & 213 (21.9) & 18.8 & $13^{*}$ \\
\hline $25-34$ & $363(27.9)$ & $237(24.3)$ & 27.9 & \\
\hline $35-44$ & $279(21.4)$ & $236(24.2)$ & 20.9 & \\
\hline $45-54$ & $212(16.3)$ & 184 (18.9) & 15.1 & $01^{*}$ \\
\hline $55-64$ & $110(8.4)$ & $90(9.2)$ & 10.6 & 0.156 \\
\hline$\geq 65$ & $22(1.7)$ & $14(1.4)$ & & $<0.001^{*}$ \\
\hline \multicolumn{5}{|l|}{ Geographical region ${ }^{b}$} \\
\hline Greater Cairo & $511(39.3)$ & $256(26.3)$ & & 0.390 \\
\hline Alexandria & $123(9.5)$ & $119(12.2)$ & 2.4 & 0.849 \\
\hline Delta & $229(17.6)$ & $202(20.8)$ & 2.7 & 0.497 \\
\hline Suez Canal & $123(9.5)$ & $114(11.7)$ & 11.2 & 0.624 \\
\hline North upper Egypt & $144(11.1)$ & $122(12.6)$ & 12.9 & 0.779 \\
\hline Asyut & $45(3.5)$ & $44(4.5)$ & 4.9 & 0.561 \\
\hline South upper Egypt & $126(9.7)$ & $115(11.8)$ & 11.8 & 1 \\
\hline \multicolumn{5}{|l|}{ Residence $^{\mathrm{b}}$} \\
\hline Urban & $934(71.8)$ & $658(67.7)$ & 42.2 & $<0.001^{*}$ \\
\hline Rural & $367(28.2)$ & $314(32.3)$ & 57.8 & $<0.001^{*}$ \\
\hline \multicolumn{5}{|l|}{ Education level ${ }^{\mathrm{b}}$} \\
\hline Illiterate & $116(8.9)$ & $9(11.2)$ & 25.8 & $<0.001^{*}$ \\
\hline Below intermediate $^{c}$ & $311(23.9)$ & $290(29.8)$ & 29.0 & 0.833 \\
\hline Intermediate $^{\mathrm{d}}$ & $511(39.3)$ & $398(40.9)$ & 29.1 & $<0.001 *$ \\
\hline University degree and above & $363(27$. & $175(18)$ & 15.5 & $<0.001^{*}$ \\
\hline \multicolumn{5}{|l|}{ Employment status ${ }^{\mathrm{b}}$} \\
\hline Employed & $950(73)$ & $728(74.9)$ & 74.4 & 0.721 \\
\hline Unemployed/retired/students/other & $351(27)$ & $244(25.1)$ & 25.6 & 0.721 \\
\hline \multicolumn{5}{|l|}{ Marital status ${ }^{b}$} \\
\hline Married & & $602(61.9)$ & 68 & $<0.001^{*}$ \\
\hline Single/divorced/widowed & $561(43.2)$ & $370(38.1)$ & 32 & $<0.001^{*}$ \\
\hline \multicolumn{5}{|l|}{ Religious beliefs ${ }^{\mathrm{b}}$} \\
\hline Muslim & $1241(95.4)$ & $931(95.8)$ & $94.9^{\mathrm{e}}$ & 0.202 \\
\hline Christian & $60(4.6)$ & $41(4.2)$ & 5.1 & 0.202 \\
\hline Presence of ch & $414(31.8)$ & $285(29.3)$ & - & - \\
\hline Covered (fv' & $786(60.4)$ & $579(59.6)$ & 54.7 & $0.0021 *$ \\
\hline No cov & $515(39.6)$ & $393(40.4)$ & 45.3 & $0.0019 *$ \\
\hline VAS & $77.5 \pm 16.2$ & $76.9 \pm 16.7$ & & \\
\hline & $893(68.5)$ & $664(68.2)$ & & \\
\hline Sligh golems & $234(18)$ & $169(17.4)$ & & \\
\hline Moderate problems & $136(10.4)$ & $107(11)$ & & \\
\hline Severe problems & $39(3)$ & $34(3.5)$ & & \\
\hline Unable to walk & $1(0.1)$ & $0(0)$ & & \\
\hline \multicolumn{5}{|l|}{ Self-care } \\
\hline No problems & $1226(94.1)$ & 912 (93.6) & & \\
\hline Slight problems & $52(4)$ & $40(4.1)$ & & \\
\hline
\end{tabular}


Table 1 (continued)

\begin{tabular}{|c|c|c|c|c|}
\hline Characteristics & Full sample $(n=1303)$ & Actual sample $(n=974)$ & $\begin{array}{l}\text { General } \\
\text { population }^{\mathrm{a}}\end{array}$ & $p$ value \\
\hline Moderate problems & $17(1.3)$ & $15(1.5)$ & & \\
\hline Severe problems & $8(0.6)$ & $7(0.7)$ & & \\
\hline Unable to dress and wash & $0(0)$ & $0(0)$ & & \\
\hline \multicolumn{5}{|l|}{ Usual activities } \\
\hline No problems & $891(68.4)$ & $667(68.5)$ & & \\
\hline Slight problems & $252(19.3)$ & $183(18.8)$ & & \\
\hline Moderate problems & $132(10.1)$ & $100(10.3)$ & & \\
\hline Severe problems & $24(1.8)$ & $21(2.2)$ & & \\
\hline Unable to do usual activities & $4(0.3)$ & $3(0.3)$ & & \\
\hline \multicolumn{5}{|l|}{ Pain/discomfort } \\
\hline No problems & $510(39.1)$ & $386(39.6)$ & & \\
\hline Slight problems & $436(33.5)$ & $302(31)$ & & \\
\hline Moderate problems & $284(21.8)$ & $219(22.5)$ & & \\
\hline Severe problems & $52(4)$ & $48(4.9)$ & & \\
\hline Extreme pain or discomfort & $21(1.6)$ & $19(2)$ & & \\
\hline \multicolumn{5}{|l|}{ Anxiety/depression } \\
\hline No problems & $420(32.2)$ & $348(35$ & & \\
\hline Slight problems & $410(31.5)$ & $287(29.5)$ & & \\
\hline Moderate problems & $343(26.3)$ & 82 & & \\
\hline Severe problems & $76(5.8)$ & $(6.3)$ & & \\
\hline Extreme anxiety or depression & $54(4.1)$ & $46(4.7)$ & & \\
\hline \multicolumn{5}{|c|}{ Data are presented as $n(\%)$, mean \pm standard deviation (range), or $\% y$ ss oth } \\
\hline \multicolumn{5}{|l|}{$V A S$ visual analogue scale } \\
\hline \multicolumn{5}{|c|}{${ }^{*} p<0.05$ (based on 1-sample z-test for a popul } \\
\hline \multicolumn{5}{|c|}{${ }^{a}$ Data estimated from the Egyptian Central Agency for Pub ${ }^{1}{ }_{1}$ zatio and Statistics, March 2019[11] } \\
\hline \multicolumn{5}{|c|}{${ }^{\mathrm{b}}$ Sample size was $n=1301$ for the full sample and $n=97>\mathrm{r}$ the actu ample } \\
\hline \multicolumn{4}{|c|}{${ }^{c}$ Below intermediate: below high school level } & ${ }^{\mathrm{d}}$ Intermediate: high school level or 2 years insti ate \\
\hline${ }^{\mathrm{e}}$ Data obtained from Mohamoud & 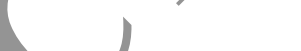 & & & \\
\hline
\end{tabular}

values increased with increasing sive, ithe health state. The heteroskedastic mode ed was a generalization of the Tobit model that uses in rval rugression (intreg) command of STATA. Th intru ommand models the error term as a function of dumm, MO2-AD5, accounting for multiplicative hetero dasticity. This means that the error term is modelled in the same way over all participants. The dependen ${ }^{\prime}$ rabl of the cTTO data was the disutility defi as I inas the cTTO observed value for a given 1 th the The DCE data were analyzed using the condition ${ }^{1}$ ogit model (model 5), where a binary outcome was used $(4,1), 0$ for dead and 1 for full health, representing the choice of the participant for each pair of the DCE tasks. To compare the modelling results of the cTTO and DCE data, the coefficients of the DCE model were rescaled using the rescaling parameter of the TTO model estimations [27, 28]. The cTTO and DCE data were combined in a hybrid model by multiplying the likelihood function of the cTTO model by the likelihood function of the DCE model [27, 28]. Four hybrid models were tested (models 6-9) by allowing heteroskedasticity, random effects, and/or censoring at -1 for the cTTO data and conditional logit model for the DCE data.

\subsection{Evaluation of the Model Performance}

The model performance was evaluated using the logical consistency of the parameter estimates, the significance level of the parameters $(p<0.05)$, the value range between observed and predicted values [27, 29], the goodness of fit using the Akaike information criteria (AIC) and Bayesian information criteria (BIC), and prediction accuracy, where root mean square error (RMSE) and mean absolute error (MAE) were calculated. Other factors were considered in model selection, such as accounting for the censored nature of the data, heteroskedasticity of the error term, and heterogeneity of the participants' views. Finally, a sensitivity analysis was 


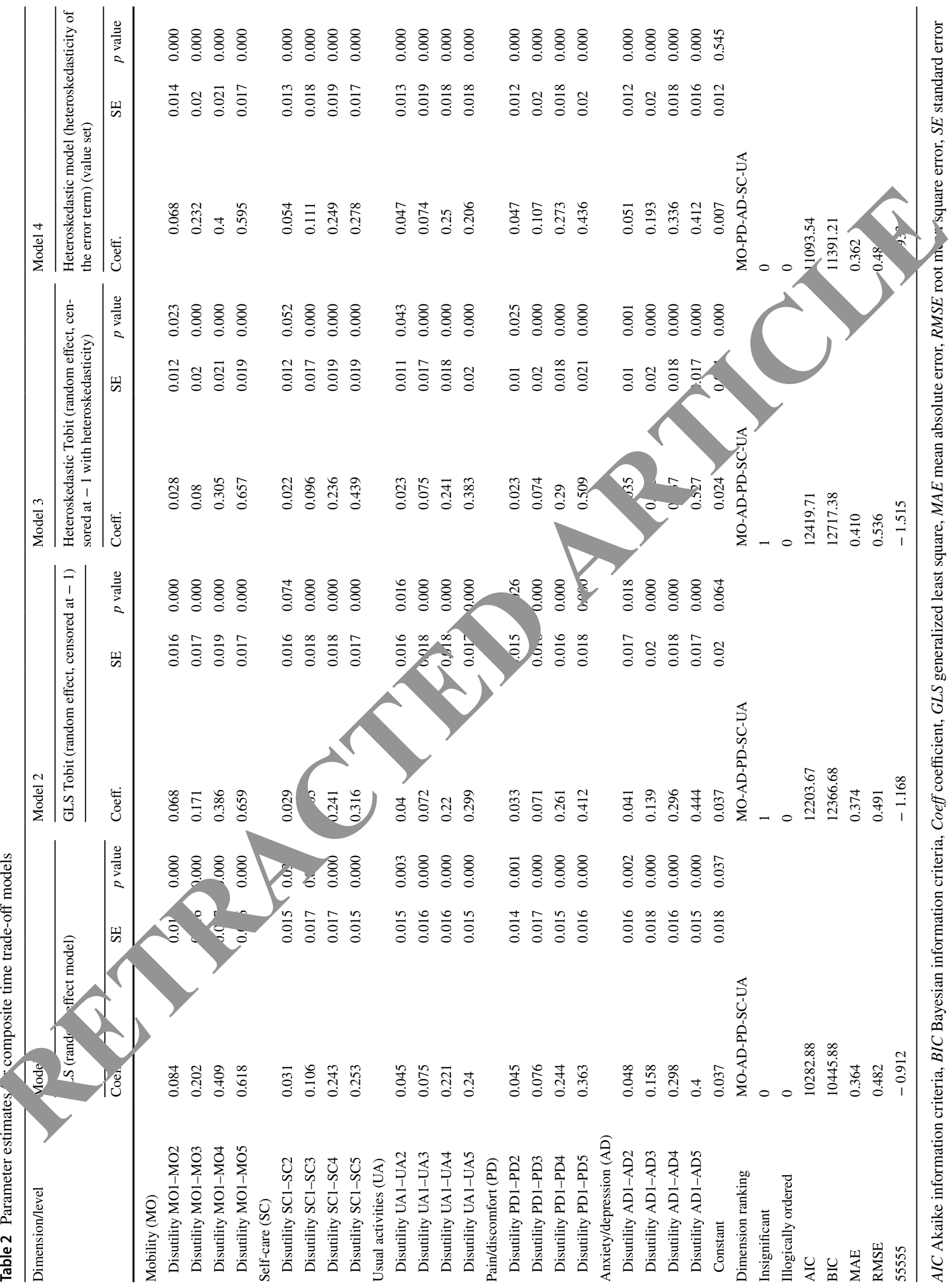


performed to evaluate the robustness of the tested models by re-inclusion of the participants' flagged health states.

\section{Results}

\subsection{Data Cleaning}

A total of 1378 interviews were conducted from July 2019 to March 2020, where three interviewers were excluded from the data collection and their interviews were dropped $(n=$ 113) because compliance with protocol was poor. Of the remaining 1267 interviews, 75 were incomplete and 216 were pilot, which resulted in 974 interviews being included in the final analysis. We planned to have 1000 final interviews, but sampling was interrupted by the global coronavirus 2019 (COVID-19) pandemic. We had good-quality data because we followed the QC criteria strictly and had an extensive pilot phase, so 974 interviews was deemed adequate.

\subsection{Participants' Characteristics}

Table 1 shows the characteristics of the study sample in comparison with the Egyptian general population [11, 30]. The average age was 36.9 years, and $52.4 \%$ of the participants were males. Overall, the sample of participants was representative of the Egyptian adult general populatior with respect to age, sex, and geographical distribution. Hou $r$, compared with national statistics, we found th + illitera participants, elderly participants ( $\geq 65$ years), anc sidents of rural areas were underrepresented in or sample, w creas those aged 35-54 years were overrepre ented.

\subsection{Self-Reported Health Usi $\sim$ the $E \times \sim 0-5 \mathrm{~L}$ Descriptive System}

In the actual sample, $13 \%$ f nart cipants were in a state of full health (1111, T, most common health problem

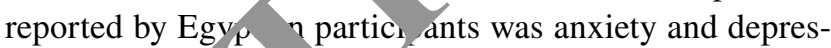
sion $(64.3 \%)$, wher the least common health problem was self-care (6.3\%). T) ie mean VAS score was $76.9 \pm 16.7$ (Table 1).

\section{Cr mnosice Time Trade-Off and Discrete-Choice} Yperiment Data

The 974 interviews provided 9740 cTTO responses and 6818 DCE responses. The mean interview time was $41 \pm 16$ min. The mean iterative steps to reach the point of indifference was $7.2 \pm 3.2$. The mean time spent in the feedback module was $2.8 \pm 10.4 \mathrm{~min}$. The participants flagged 898 cTTO responses using the feedback module. A total of 254 (26\%)
Table 3 Parameter estimates for discrete-choice experiment model

\begin{tabular}{|c|c|c|c|c|}
\hline \multirow[t]{3}{*}{ Dimension/level } & \multicolumn{4}{|c|}{ Model 5} \\
\hline & \multicolumn{4}{|c|}{ Conditional logit model } \\
\hline & Beta & Res & SE & value \\
\hline \multicolumn{5}{|l|}{ Mobility (MO) } \\
\hline Disutility MO1-MO2 & 0.266 & 0.069 & 0.058 & 0.000 \\
\hline Disutility MO1-MO3 & 0.36 & 0.093 & $0.06^{\circ}$ & 000 \\
\hline Disutility MO1-MO4 & 1.13 & 0.291 & & \\
\hline Disutility MO1-MO5 & 2.286 & 0.587 & & \\
\hline \multicolumn{5}{|l|}{ Self-care (SC) } \\
\hline Disutility SC1-SC2 & 0.241 & 0 & & 0 \\
\hline Disutility SC1-SC3 & 0.237 & & & 0.001 \\
\hline Disutility SC1-SC4 & 0.783 & & 0.071 & 0.000 \\
\hline Disutility SC1-SC5 & 1. & & 0.074 & 0.000 \\
\hline \multicolumn{5}{|l|}{ Usual activities (UA) } \\
\hline Disutility UA1-UA2 & & & 0.06 & 0.000 \\
\hline Disutility UA1-UA3 & 0. & 0.067 & 0.069 & 0.000 \\
\hline Disutility UA & 0.837 & 0.215 & 0.07 & 0.000 \\
\hline Disutility UA & 1548 & 0.399 & 0.076 & 0.000 \\
\hline \multicolumn{5}{|l|}{ Pain/discomfort (PL } \\
\hline sutility & 0.18 & 0.046 & 0.064 & 0.005 \\
\hline Disutility PD & 0.273 & 0.07 & 0.069 & 0.000 \\
\hline Disutility P1 1-PD4 & 0.734 & 0.189 & 0.069 & 0.000 \\
\hline tility PD1-PD5 & 1.435 & 0.369 & 0.075 & 0.000 \\
\hline xic /depression (AD & & & & \\
\hline isv ility AD1-AD2 & 0.08 & 0.02 & 0.065 & 0.222 \\
\hline Lisutility AD1-AD3 & 0.25 & 0.064 & 0.067 & 0.000 \\
\hline Disutility AD1-AD4 & 0.823 & 0.212 & 0.074 & 0.000 \\
\hline Disutility AD1-AD5 & 1.529 & 0.394 & 0.082 & 0.000 \\
\hline Ranking of dimensions & \multicolumn{4}{|c|}{ MO-UA-AD-SC-PD } \\
\hline Insignificant & \multicolumn{4}{|l|}{1} \\
\hline Illogically ordered & \multicolumn{4}{|l|}{2} \\
\hline AIC & \multicolumn{4}{|c|}{6776.675} \\
\hline $\mathrm{BIC}$ & \multicolumn{4}{|c|}{6913.222} \\
\hline 55555 & \multicolumn{4}{|c|}{-1.135} \\
\hline
\end{tabular}

$A I C$ Akaike information criteria, BIC Bayesian information criteria, $S E$ standard error

participants had at least one inconsistency, which reduced to $122(12.5 \%)$ after using the feedback module. The number of inconsistencies related to severity ' 6 ' (mild issue in one dimension only) and 55555 states were $11(1 \%)$ and 31 (3\%), respectively, which reduced to $6(0.6 \%)$ and $3(0.3 \%)$, respectively, after using the feedback module.

The main analysis included all the unflagged cTTO valuations ( 8842 responses); $41 \%$ of these were considered WTD, and the mean observed value was negative for 36 of the 86 health states included in the cTTO design. The percentages of values clustered at $-1,-0.5,0,0.5$, and 1 were $13.3 \%, 4 \%, 1.5 \%, 5.2 \%$, and $12.3 \%$, respectively (Fig. 1). As the level sum score increased for the 


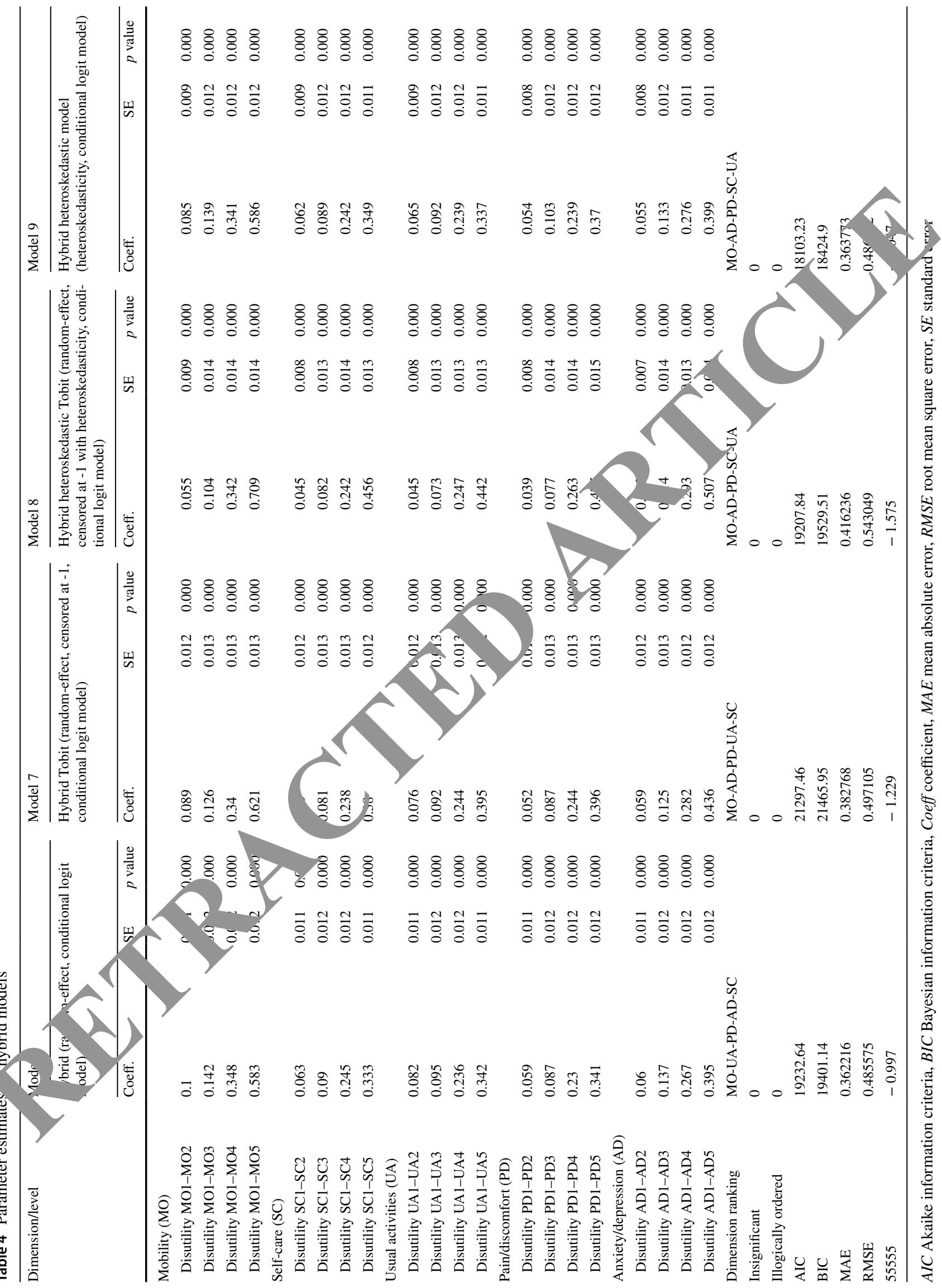


EQ-5D-5L health states, lower mean TTO values and a larger standard deviation were observed (Fig. 2). The mean observed cTTO values of the 86 health states was $0.12 \pm 0.73$, which ranged from $0.96 \pm 0.08$ for health state 11211 to $-0.83 \pm 0.3$ for health state 55555 .

For the DCE tasks, the participants were more likely to choose the health states with the lower misery score as the difference in severity increased between the two health states. In total, 23 participants (2.4\%) answered using the following specific pattern (AAAAAAA, BBBBBBB, ABABABA, BABABAB). However, their mean time to complete the DCE tasks was acceptable, so we decided not to exclude these interviews from the analysis.

\subsection{Modelling Results}

Modelling results are shown for cTTO, DCE and hybrid models in Tables 2, 3 and 4 respectively. All the tested models were logically consistent except for some minor inconsistencies appearing in the conditional logit models for DCE data in the self-care and usual activities dimensions, level 3 (SC3 and UA3) (Table 3). Furthermore, all model parameter estimates were statistically significant except self-care dimension level 2 (SC2) for the Tobit and heteroskedastic Tobit models (models 2 and 3 ) (Table 2) and anxiety/depression dimension level 2 (AD2) in the conditional logit model (model 5). Dimension ranking for the cTTO models in terms of relative importance were as follows. For models 1,2 and 3 , mobility was the most important dimension, follow anxiety/depression, pain/discomfort, self-care and usu. activities (least important). For the heterosk das model (model 4), pain/discomfort was more important tha inxiety/depression ( 0.436 vs. 0.412 , respect ely). Disutility values of the DCE model (model 5) were ca lated by dividing the coefficients of the DCE moc ${ }^{-1}$ by the rescaling factor (factor $=3.884$ ). Mobility had the la ${ }_{0}$ impact on health state preference values for the tested models.

\subsection{Preferred Mcial a. Value Set}

Both the GLS and $\mathrm{L}$ heteroskedastic models performed better thar the other te, red models in terms of logical consistency, and rictio curacy (MAE and RMSE) (Table 2). How$f r$ th heteroskedastic model (model 4) was considered the ferred model because of its ability to handle the variability of the error term. In addition, it had a lower MAE than the other tested models, indicating better accuracy. The constant term in the model was not significant and reported a reasonable value range between the observed and predicted values (Fig. 3).

The predicted cTTO values ranged from -0.933 for the worst health state (55555) to 0.953 for 11211 and 11121 .
About 36.3\% (1136) of the health states were WTD. Dimension ranking in terms of relative importance was mobility (most important), pain/discomfort, anxiety/depression, selfcare, and usual activities (least important). For any given health state, the utility value can be calculated by subtracting the regular dummies (parameter estimates) for each dimension level of the health state from 1 .

\subsection{Sensitivity Analysis}

The model performance worsened after the inclusio $f$, ne flagged health states in the feedback mo so we decided to exclude the flagged health states fom $\mathrm{t}$ ana ysis. No other exclusions were applied to th data as only three inconsistencies related to 55555, and oni one par icipant gave the same value for all health statc

\section{Discussicn}

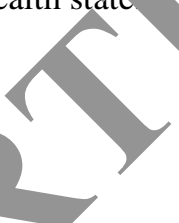

To the best of our owledge, this is the first EQ-5D-5L valuation s.. in Egypt and in the Middle East and North Africa (MANA) region. A consistent tariff was generated $w^{-1}$ h statisti ally significant decrements for all dimensions that $n$ be used as a scoring system for economic evaluain, hform decision making, and improve the quality of $F_{1} A$ in the Egyptian healthcare system.

The successful application of the EQ-VT valuation protocol on the Egyptian population verified the feasibility and cultural appropriateness of using such valuation techniques in Muslim and Arabic-speaking countries. Furthermore, the extensive pilot phase and the periodic QC meetings allowed the Egyptian study team and the EQ-VT support team to enhance the interviewers' performance and promote compliance with the valuation tasks.

The heteroskedastic model (model 4) based on the cTTO data was selected as the preferred model for the Egyptian tariff. The cTTO data were of very good quality, as all the parameter estimates of all the tested models were logically consistent and statistically significant and only two inconsistencies appeared in the DCE conditional logit model. Furthermore, in the tested models, there was a large difference in terms of size of coefficients for the five dimensions at different levels for the DCE and TTO data as both techniques have different underlying assumptions. cTTO data are timedependent data influenced by scale compatibility and loss aversion [20, 31], whereas the DCE is a choice-based task characterized by attribute non-attendance and lexicographic preferences [32, 33]. Other countries, such as the USA [34], the Netherlands [35], China [36], Uruguay [37], Korea [38], and Hungary [39] also used only cTTO data to generate their national value set. 
Fig. 1 Observed (composite time trade-off [cTTO]) value distribution

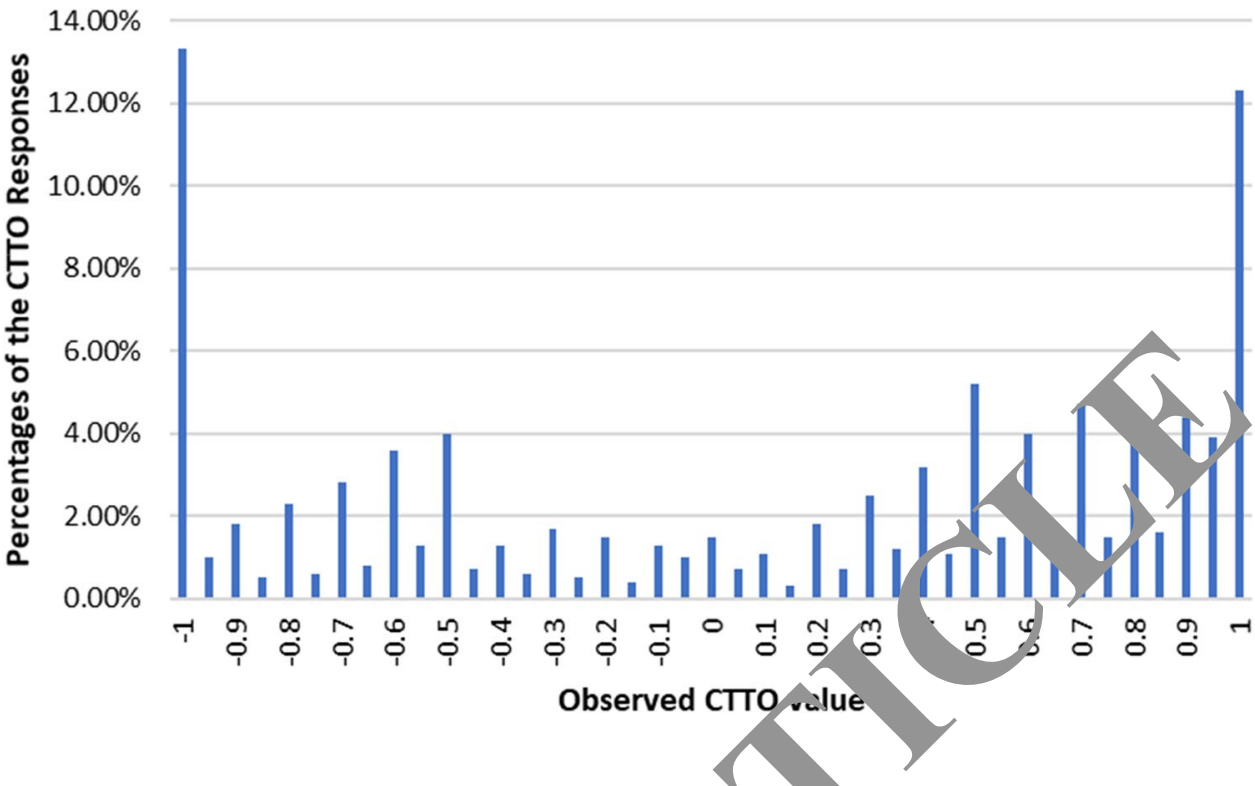

All EQ-5D-5L valuation studies followed the same standardized international protocol (EQ-VT) so the results can be easily compared across countries. In Egypt, mobility had the largest impact on health state preference values. This may be due to limited access to social welfare for immobility. Furthermore, Egypt lacks the infrastructure that enables people with mobility problems to live normally and independently in society. Mobility was also the most important dimension in all Asian countries [36, 38, 40-46], Hungary [39], Un'guay [37], and Canada [47].

In this study, the predicted cTTO values ranced $\mathrm{n}$ -0.933 for the worst health state (55555) to 0.95 for 112 . and 11121. The worst health state had a hig. er ve than in Taiwan (-1.0259) [42] and Ireland $(-0.974)[$. 3 but was lower than all other published valu: ion studies [34-41, 43-47, 49-54].

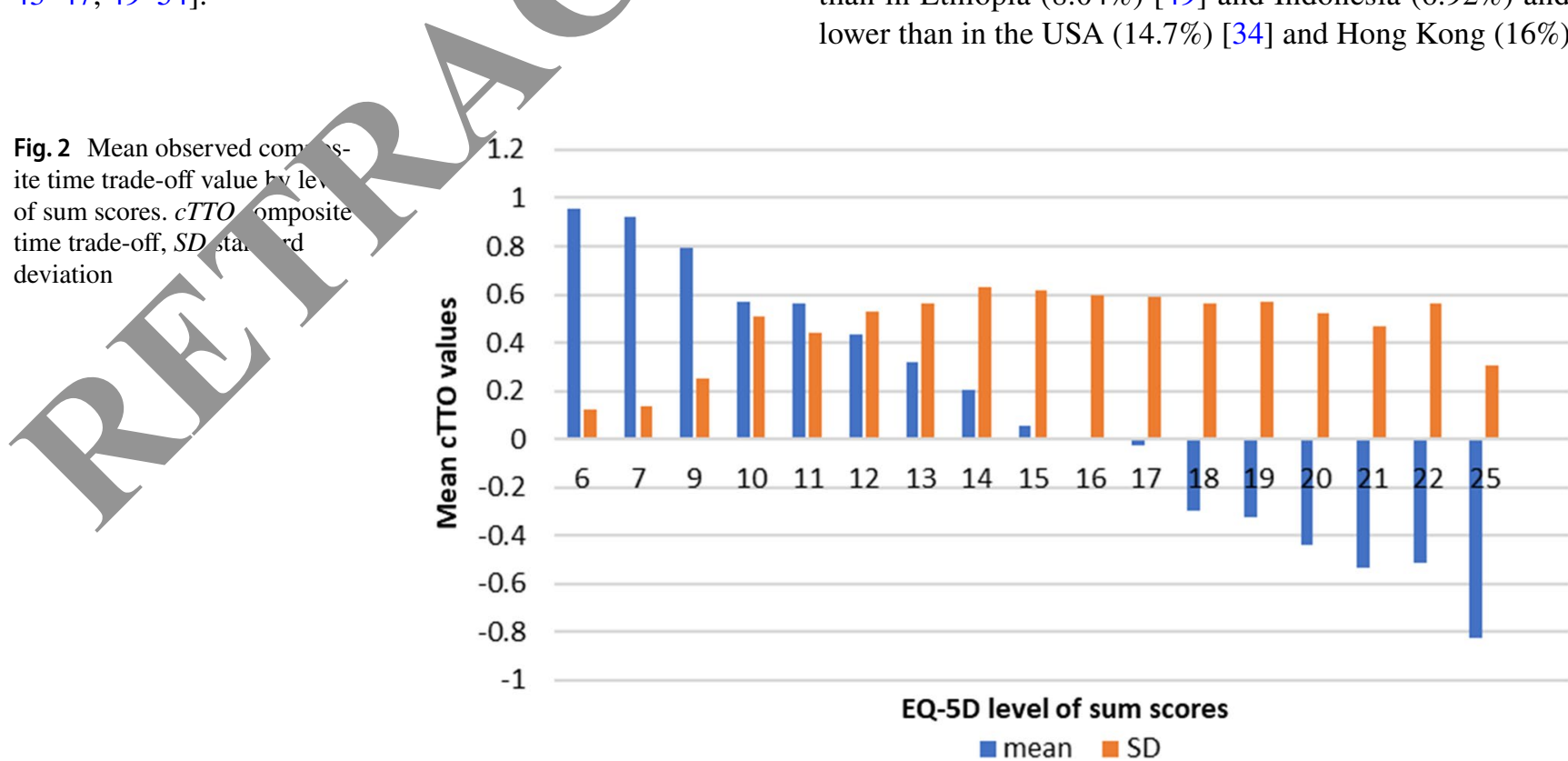

Egypt had the laroest per tage (41\%) of cTTO observations considere to WTD compared with other countries such as Taiwan $0 . . n, 442]$, Hong Kong and Indonesia (36\%) [40 46], Jap and Korea $(0.1 \%)$ [38, 45], The Netherlands (2\%, China (10\%) [36], and Ethiopia (11.7\%) [49]. This ray oe attributed to cultural and social factors most par Cipants in Egypt preferred to die than to be a bur on family and friends if they had severe illness, as tatec in the country-specific questionnaire (publication of a aled questionnaire results is underway). This is in line with findings published in the Indonesian EQ-5D-5L valuation study [40].

There were 1172 (13.3\%) observations at -1 , where the participants traded all 20 years of life to avoid living in certain health states in the cTTO task; this percentage is higher than in Ethiopia (8.04\%) [49] and Indonesia (6.92\%) and lower than in the USA (14.7\%) [34] and Hong Kong (16\%) mean $\quad \mathrm{SD}$ 
[46]. Furthermore, 12.3 and $1.5 \%$ of the observations were clustered at 1 and 0 , respectively, compared with 20.5 and $5.1 \%$, respectively, in the USA [34]. Clustering at these critical points might be due to interviewer's effect, task shortcutting, and social and cultural factors. In this study, the QC tool was used rigorously and the pilot phase was extensive to reduce the variability among and within interviewers, standardize their performance, and improve data quality.

There were some limitations in terms of differences in the distribution of background variables in the actual sample compared with the data provided by the Egyptian Central Agency for Public Mobilization and Statistics [11]. Rural and illiterate participants were underrepresented in our sample, it was difficult for the interviewers to reach some rural areas but extreme effort was made to represent people living in those areas as much as possible; however, the sample accurately represented the geographical distribution in Egypt. The EQ-VT protocol was designed for literate and educated participants. Tunisia recently published an EQ5D-3L valuation study that only included literate individuals, in spite of the fact that illiterate people represent $18.8 \%$ of the general Tunisian population [55]. However, our study team decided not to exclude illiterate participants from the Egyptian study to ensure they had a voice in the produced tariff. The team exerted all possible efforts to interview illiterate participants with the use of some visual aids, but decided not to follow the exact quota for illiterate partiripants (25.8\%) because the tool used was not fully valj ated.

Other demographic characteristics are shown in $\mathrm{Ta}$ Some characteristics did not significantly d c iate fro. the population, such as religion and emplo mo status, whereas marital status and health insurnce covera differed significantly from the population istribution. Despite the deviations from the exact populat distribution, the demographic characteristics st " nroducen the required diversity. Furthermore, the estimate sta was not fully

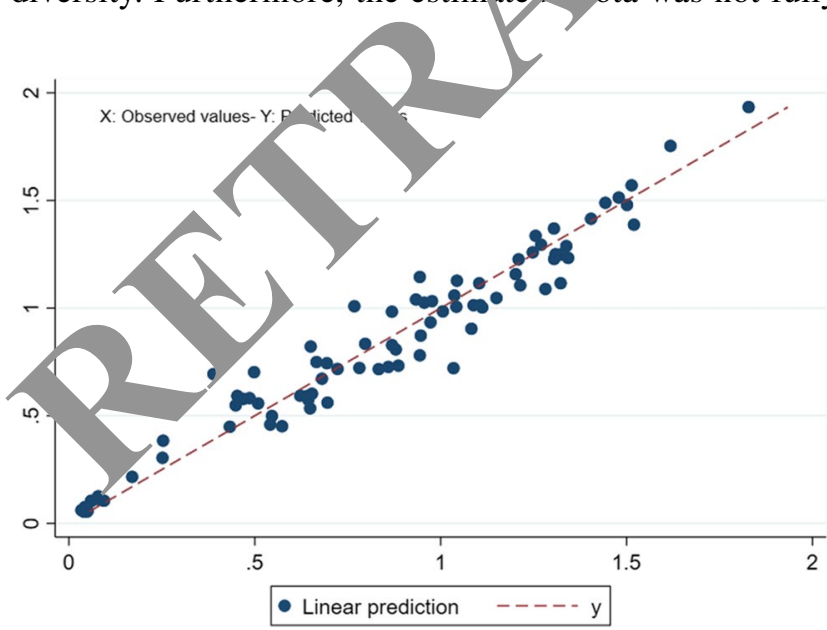

Fig. 3 Scatterplots of the predicted values of the heteroskedastic model versus observed values of composite time trade-off fulfilled because the COVID-19 pandemic led to the sudden interruption of the data collection. These deviations in the sample characteristics in terms of residence and/or education are in line with other valuation studies. Further research is needed to assess the feasibility and impact of weighting of underrepresented characteristics on the produced value sets. To explore how demographic characteristics affected participants' preferences, a publication exploring the effect of cultural and demographic differences on healtb valuation in Egypt is underway.

Finally, the availability of the Egyptian tariff will coarage health economists and clinicians to lude qialiny-oflife questionnaires in clinical trials and it ement costutility analysis and pharmacoecon mic mode, ng to assist decision makers in appropriate 'ocatio of healthcare resources.

Since cultural and so 10econ ic,factors play a role in shaping people's prefer es, the nigh quality of the data used in the Egyptian valuc may allow its use in economic evaluati ns to MENA countries that share common cultural and so a nic backgrounds but for which a country-cnecific $v_{c} \approx$ set is not yet available, rather than using tari $n$ gutside the region [56]. It must be noted that recompencations are for each country to develop its value $s, \mu$ to represent the views and preferences of its own opulation [57].

\section{Conclusion}

This is the first value set for EQ-5D-5L based on social preferences obtained from a nationally representative sample in Egypt. The value set will play a key role in economic evaluations and HTAs in Egypt. In addition, other countries in the MENA region may be encouraged to follow suit and develop their own value sets.

Acknowledgements The authors thank the EuroQol support teamElly Stolk, Fatima Al Sayah, and Arnd Jan Prause-for their guidance and support during study preparation, data collection, and QC. Special thanks to Bram Roudijk for providing support in the analysis of the valuation data and interpretation of results. We are grateful for the outstanding effort of the interviewers: Eglal A. Bassiouny, Hend K. Eldeib, Sandra N. Naguib, Israa K. Mohamed, Sara AlSherif, Shaza G. Ali, Salma M. Abdelmageed, and Nour A. Sharaf. Finally, we thank the study participants for taking part in this study.

\section{Declarations}

Funding This project received financial support from Bournemouth University and the EuroQol Foundation, the Netherlands (project ID: 20180130). The funding agreements ensured the authors' independence in designing the study and writing and publishing the results. 
Conflict of Interest Aureliano Paolo Finch is a member of the EuroQol Research Foundation (the copyright holder of the EQ-5D-5L). Sahar A. Al Shabasy, Maggie M. Abbassi, Darrin Baines, and Samar F. Farid have no conflicts of interest directly relevant to the content of this article.

Availability of Data and Material The datasets generated and analyzed during the current study are available from the corresponding author upon reasonable request.

Ethics Approval The study received ethical approval from the Ethics Committee of the Faculty of Pharmacy, Cairo University, and was conducted in accordance with the Declaration of Helsinki.

Consent to Participate Written informed consent was obtained from all participants included in the study. Participants were informed about their freedom of refusal. Anonymity and confidentiality were maintained throughout the research process.

Author Contributions SAAS participated in the study preparation and data collection, created data QC reports, interpreted results, and prepared the draft manuscript. MMA and SFF participated in the study preparation, proof reading of the translated version, follow-up of data collection, the QC process, interpreting results, and reviewing the final manuscript. APF participated in follow-up of data collection, the QC process, statistical analysis and interpretation of the study results, and review of the final manuscript. Darrin Baines participated in the study preparation, acquired the funding, and reviewed the final manuscript.

Open Access This article is licensed under a Creative Commons Attribution-NonCommercial 4.0 International License, which permits any non-commercial use, sharing, adaptation, distribution and reproduction in any medium or format, as long as you give appropriate credit to 'he original author(s) and the source, provide a link to the Creative commons licence, and indicate if changes were made. The images 0 third party material in this article are included in the artic'e s Crea Commons licence, unless indicated otherwise in a cr. line to th material. If material is not included in the article's Crative mmons licence and your intended use is not permitted oy statutory gulation or exceeds the permitted use, you will ne d to obtain permission directly from the copyright holder. To view a c y of this icence, visit http://creativecommons.org/licenses/by-nc/4.0

\section{References}

1. Brooks R, Grou EuroQ 1 : the current state of play. Health Policy. 1996;37(1). 72.

2. Brauer CA, Rosen Ab Greenberg D, Neumann PJ. Trends in the meas me to health utilities in published cost-utility analyses. Value $\mathrm{H}_{\mathrm{t}}$ th. 20 ,6;9(4):213-8.

3. 1. er L, enberg E (eds). Trends in utility elicitation methods is there still a role for direct elicitation. In: 32nd Annual

eetms of Society for Medical Decision Making, Toronto; 2010.

4. $\mathrm{k}$ R, Charro Fd. EQ-SD: a measure of health status from the EuroQol Group. Ann Med. 2001;33(5):337-43.

5. Kennedy-Martin M, Slaap B, Herdman M, van Reenen M, Kennedy-Martin T, Greiner W, et al. Which multi-attribute utility instruments are recommended for use in cost-utility analysis? A review of national health technology assessment (HTA) guidelines. Eur J Health Econ. 2020;21(8):1245-57.

6. Herdman M, Gudex C, Lloyd A, Janssen M, Kind P, Parkin $\mathrm{D}$, et al. Development and preliminary testing of the new five-level version of EQ-5D (EQ-5D-5L). Qual Life Res. 2011;20(10):1727-36.

7. The EQ-5D Instruments. 2020. https://euroqol.org/eq-5d-instr uments/. Accessed 2 Dec 2020.

8. Janssen M, Pickard AS, Golicki D, Gudex C, Niewada M, Scalone $\mathrm{L}$, et al. Measurement properties of the EQ-5D-5L compared to the EQ-5D-3L across eight patient groups: a multi-country study. Qual Life Res. 2013;22(7):1717-27.

9. Janssen MF, Bonsel GJ, Luo N. Is EQ-5D-5L better than EQ5D-3L? A head-to-head comparison of descriptive systems and value sets from seven countries. Pharmacofconomics. 2018;36(6):675-97.

10. Devlin N, Brazier J, Pickard AS, Stolk E. 3L, 5L, Wh. re L? A NICE Conundrum. PharmacoEconomics. 2018;36(6):63 0.

11. Central Agency for Public Mobilization a Statisti $\mathrm{s}-\mathrm{CAP}$ MAS. 2019. http://www.capmas.o. eg/ es/S laticPage s.aspx?page_id=5035. Accessed 9 lay 2019.

12. Kausch K. Egypt: Inside-out. Geo litics and democracy in the Middle East. FRIDE; 2015:21/34 c

13. Elsisi GH, Kaló Z, Eldessoúk1 Glmàrunvy MD, Saad A, Ragab $\mathrm{S}$, et al. Recommendatio is for rep ing pharmacoeconomic evaluations in Egypt. Va a alth Reg ssues. 2013;2(2):319-27.

14. Elsisi G, Hassouna $A$ Tal $A$, Elmahdawy M, Ibrahim S. Costeffectiveness of opanib sus sunitinib in egyptian patients with metast ic re al cell carcinoma from the health insurance perspective: odel. Value Health. 2014;17(3):A90-1.

15. Elsisi G, Abda HM, Elmansy H. Economic evaluation of lidoca 'tetracain patch versus lidocaine/prilocaine cream for topical ana of before vascular access in Egypt. Value Health. 2015;18 1):A669.

16 El-Ham: Ísy MH, Elsisi GH, Eldessouki R, Elmazar MM, ha AS, Awad BF, et al. Economic evaluation of the combined : of warfarin and low-dose aspirin versus warfarin alone in nechanical valve prostheses. Appl Health Econ Health Policy. 2016;14(4):431-40

17. Elsisi GH, Eldessouki R, Kalo Z, Elmazar MM, Taha AS, Awad $\mathrm{BF}$, et al. Cost-effectiveness of the combined use of warfarin and low-dose aspirin versus warfarin alone in Egyptian patients with aortic valve replacements: a Markov model. Value Health Reg Issues. 2014;4C:24-30.

18. Hossam M, Elsisi G. Cost-effectiveness analysis of fidaxomicin versus oral vancomycin for the treatment of clostridium difficile infection in Egypt. Value Health. 2016;19(7):A513.

19. Mostafa A, Elsisi GH. A cost-effectiveness analysis of the use of safety-engineered syringes in reducing HBV, HCV, and HIV burden in Egypt. Expert Rev Med Devices. 2019;16(2):155-63.

20. Oppe M, Devlin NJ, van Hout B, Krabbe PF, de Charro F. A program of methodological research to arrive at the new international EQ-5D-5L valuation protocol. Value Health. 2014;17(4):445-53.

21. Xie F, Pickard AS, Krabbe PF, Revicki D, Viney R, Devlin N, et al. A checklist for reporting valuation studies of multi-attribute utility-based instruments (CREATE). PharmacoEconomics. 2015;33(8):867-77.

22. Oppe M, Van Hout B. The "power" of eliciting EQ-5D-5L values: the experimental design of the EQ-VT. EuroQol Working Paper Series. 2017;17003. http://euroqol.org/wp-content/uploa ds/2016/10/EuroQol-Working-Paper-Series-Manuscript-17003 -Mark-Oppe.pdf. Accessed 10 May 2020.

23. Oppe M, Rand-Hendriksen K, Shah K, Ramos-Goñi JM, Luo N. EuroQol protocols for time trade-off valuation of health outcomes. PharmacoEconomics. 2016;34(10):993-1004.

24. Devlin NJ, Tsuchiya A, Buckingham K, Tilling C. A uniform time trade off method for states better and worse than dead: feasibility study of the 'lead time'approach. Health Econ. 2011;20(3):348-61. 
25. General Organization for Physical Planning. http://gopp.gov.eg/ eg-map/. Accessed April 22018.

26. Ramos-Goñi JM, Oppe M, Slaap B, Busschbach JJ, Stolk E. Quality control process for EQ-5D-5L valuation studies. Value Health. 2017;20(3):466-73.

27. Ramos-Goñi JM, Pinto-Prades JL, Oppe M, Cabasés JM, Serrano-Aguilar P, Rivero-Arias O. Valuation and modeling of EQ-5D-5L health states using a hybrid approach. Med Care. 2017;55(7):e51-8.

28. Ramos-Goni JM, Craig B, Oppe M, van Hout B. Combining continuous and dichotomous responses in a hybrid model. EuroQol Working Paper Series. 2016;16002. https://euroqol.org/wpcon tent/uploads/working_paper_series/EuroQol_Working_Paper _Series_Manuscript_16002_-_Juan_Ramos-Goni.pdf. Accessed 30 Apr 2020.

29. Feng Y, Devlin NJ, Shah KK, Mulhern B, Hout B. New methods for modelling EQ-5D-5L value sets: an application to English data. Health Econ. 2018;27(1):23-38.

30. Mohamoud YA, Cuadros DF, Abu-Raddad LJ. Characterizing the Copts in Egypt: Demographic, socioeconomic and health indicators. Q Sci Connect. 2013. https://doi.org/10.5339/conne ct.2013.22.

31. Bleichrodt $\mathrm{H}$. A new explanation for the difference between time trade-off utilities and standard gamble utilities. Health Econ. 2002;11(5):447-56.

32. Lancsar E, Louviere J. Deleting 'irrational' responses from discrete choice experiments: a case of investigating or imposing preferences? Health Econ. 2006;15(8):797-811.

33. Alemu MH, Mørkbak MR, Olsen SB, Jensen CL. Attending to the reasons for attribute non-attendance in choice experiments. Environ Resour Econ. 2013;54(3):333-59.

34. Pickard AS, Law EH, Jiang R, Pullenayegum E, Shaw JW, Xie F, et al. United States valuation of EQ-5D-5L health states using an international protocol. Value Health. 2019;22(8):931-41.

35. Versteegh MM, Vermeulen KM, Evers SM, De Wit GA, $\mathrm{R}$, Stolk EA. Dutch tariff for the five-level version of $\mathrm{E}$ Value Health. 2016;19(4):343-52.

36. Luo N, Liu G, Li M, Guan H, Jin X, Rand-Au iksen K Estimating an EQ-5D-5L value set for China. Valı. Yealth. 2017;20(4):662-9.

37. Augustovski F, Rey-Ares L, Irazola V, aray OU, Gianneo O, Fernández G, et al. An EQ-5D-5L value based o I Uruguayan population preferences. Qual Life Res. 20 $323-33$.

38. Kim S-H, Ahn J, Ock M, Sh Park J, Luo N, et al. The EQ-5D-5L valuation study in w $c$ a. Qual Life Res. 2016;25(7):1845-52.

39. Rencz F, Brodszky V, rlács L, Goli Ki D, Ruzsa G, Pickard AS, et al. Parallel valuation $\quad D-3 \mathrm{~L}$ and EQ-5D-5L by time trade-off in Hun ary. Valu ealth. 2020;23(9):1235-45.

40. Purba FD, Hy JA, Iska darsyah A, Fitriana TS, Sadarjoen SS, Ramos-Coñi $J$ al. The Indonesian EQ-5D-5L value set. Pharma oEconomics. J17;35(11):1153-65.

41. Patta he $\mathrm{i}$ J, Thavorncharoensap M, Ramos-Goñi JM, Tongsiri $\mathrm{S}$, Ings vang $/$, Teerawattananon Y. The EQ-5D-5L valuation in 1 and. Expert Rev Pharmacoecon Outcomes Res. 20) $\cdot 18(5) \% 51-8$.
42. Lin H-W, Li C-I, Lin F-J, Chang J-Y, Gau C-S, Luo N, et al. Valuation of the EQ-5D-5L in Taiwan. PLoS ONE. 2018;13(12):e0209344.

43. Mai VQ, Sun S, Minh HV, Luo N, Giang KB, Lindholm L, et al. An EQ-5D-5L value set for Vietnam. Qual Life Res. 2020;29(7):1923-33.

44. Shafie AA, Thakumar AV, Lim CJ, Luo N, Rand-Hendriksen K, Yusof FAM. EQ-5D-5L valuation for the Malaysian population. PharmacoEconomics. 2019;37(5):715-25.

45. Shiroiwa T, Ikeda S, Noto S, Igarashi A, Fukuda T, Saito S, et al. Comparison of value set based on DCE and/or ITO data: scoring for EQ-5D-5L health states in Japan. $\mathrm{e}$ Tealth. 2016;19(5):648-54.

46. Wong EL, Ramos-Goni JM, Cheung AW, Wońg AY, Rive. irias $\mathrm{O}$. Assessing the use of a feedback modu model IQ $5 \mathrm{D}-5 \mathrm{~L}$ health states values in Hong Kong. Pati t Pat Cer.ered Outcomes Res. 2018;11(2):235-47.

47. Xie F, Pullenayegum E, Gaebel K, F nsback N, Bryan S, Ohinmaa A, et al. A time trade-off-der 'ed $v$ set of the EQ-5D-5L for Canada. Med Care. 2016;54(1,

48. Hobbins A, Barry L, K 1leher D, ah K, Devlin N, Goni JMR, et al. Utility values for th states 1 Ireland: a value set for the EQ-5D-5L. PharmaıoEco nics. 2018;36(11):1345-53.

49. Welie AG, Ge kle GB, colk E, Mukuria C, Krahn MD, Enquoselas $>\mathrm{F}$, e al. Valuing health state: an EQ-5D-5L value set for Ethiop Aealth Reg Issues. 2020;22:7-14.

50. Andrade LF, Lc ig, K, Goni JMR, Oppe M, de Pouvourville G. A value set for the EQ-5D-5L. PharmacoEconomics. $2020 ; 33(4-25$.

51. Ferreira PL, Antunes P, Ferreira LN, Pereira LN, Ramos-Goñi JM. A hy fid modelling approach for eliciting health state prefences: the Portuguese EQ-5D-5L value set. Qual Life Res. 19;28(12):3163-75.

Colicki D, Jakubczyk M, Graczyk K, Niewada M. Valuation of EQ-5D-5L health states in Poland: the first EQ-VT-based study in Central and Eastern Europe. PharmacoEconomics. 2019;37(9):1165-76.

53. Ludwig K, von der Schulenburg J-MG, Greiner W. German value set for the EQ-5D-5L. PharmacoEconomics. 2018;36(6):663-74.

54. Ramos-Goñi JM, Craig BM, Oppe M, Ramallo-Fariña Y, PintoPrades JL, Luo N, et al. Handling data quality issues to estimate the Spanish EQ-5D-5L value set using a hybrid interval regression approach. Value Health. 2018;21(5):596-604.

55. Chemli J, Drira C, Felfel H, Roudijk B, Al Sayah F, Kouki $\mathrm{M}$, et al. Valuing health-related quality of life using a hybrid approach: Tunisian value set for the EQ-5D-3L. Qual Life Res. 2021. https://doi.org/10.1007/s11136-020-02730-z.

56. Bailey $\mathrm{H}$, Kind P. Preliminary findings of an investigation into the relationship between national culture and EQ-5D value sets. Qual Life Res. 2010;19(8):1145-54.

57. Roudijk B, Donders ART, Stalmeier PF. Cultural values: can they explain differences in health utilities between countries? Med Decis Making. 2019;39(5):605-16. 